\title{
Level Set Method for Shape and Topology Optimization of Contact Problems
}

\author{
Andrzej Myśliński \\ Systems Research Institute, ul. Newelska 6, 01-447 Warsaw, Poland \\ myslinskeibspan.waw.pl
}

\begin{abstract}
This paper deals with simultaneous topology and shape optimization of elastic contact problems. The structural optimization problem for an elastic contact problem is formulated. Shape as well as topological derivatives formulae of the cost functional are provided using material derivative and asymptotic expansion methods, respectively. These derivatives are employed to formulate necessary optimality condition for simultaneous shape and topology optimization and to calculate a descent direction in numerical algorithm. Level set based numerical algorithm for the solution of this optimization problem is proposed. Numerical examples are provided and discussed.
\end{abstract}

\section{Introduction}

The paper is concerned with the formulation of a necessary optimality condition and the numerical solution of a structural optimization problem for an elastic body in unilateral contact with a rigid foundation. The contact with a given friction, described by Coulomb law, is assumed to occur at a portion of the boundary of the body. The displacement field of the body in unilateral contact is governed by an elliptic variational inequality of the second order. The results concerning the existence, regularity and finite-dimensional approximation of solutions to contact problems are given in [10]. The structural optimization problem for the elastic body in contact consists in finding such topology as well as such shape of the boundary of the domain occupied by the body that the normal contact stress along a contact boundary is minimized. It is assumed that the volume of the body is bounded.

Shape optimization of static elastic contact problems is considered, among others, in [10:20], where necessary optimality conditions, results concerning convergence of finite-dimensional approximation and numerical results are provided. Material derivative method is employed in monograph [20] to calculate the sensitivity of solutions to contact problems as well as the derivatives of domain depending functionals with respect to variations of the boundary of the domain occupied by the body. Necessary optimality conditions for shape optimization of elastic contact problems are formulated also in monograph [15]. In this monograph contact problems are considered in the mixed variational formulation and the results of numerical experiments are reported. Shape optimization of a dynamic contact problem with Coulomb friction and heat flow is considered in [13]. In this paper the material derivative method is employed to formulate

A. Korytowski et al. (Eds.): System Modeling and Optimization, IFIP AICT 312, pp. 397 410, 2009.

(c) IFIP International Federation for Information Processing 2009 
a necessary optimality condition. The finite element method for the spatial derivatives and the finite difference method for the time derivatives are employed to discretize the optimization problem. The level set based method is applied to find numerically the optimal solution.

Topology optimization deals with the optimal material distribution within the body resulting in its optimal shape [22]. The topological derivative is employed to account variations of the solutions to state equations or shape functionals with respect to emerging of small holes in the interior of the domain occupied by the body. The notion of the topological derivative and results concerning its application in optimization of elastic structures are reported in the series of papers [2 6 7 79 |14|21|22 23]. Among others, paper [23] deals with the calculation of topological derivatives of solutions to Signorini and elastic contact problems. Asymptotic expansion method combined with transformation of energy functional are employed to calculate these derivatives. Simultaneous shape and topology optimization of Signorini and elastic contact problems with or without friction are analyzed in papers [6] and [14], [16] respectively. In these papers the level set method is incorporated in the numerical algorithm.

In structural optimization the level set method [4]12 19]25] is employed in numerical algorithms for tracking the evolution of the domain boundary on a fixed mesh and finding an optimal domain. This method is based on an implicit representation of the boundaries of the optimized structure. A level set model describes the boundary of the body as an isocontour of a scalar function of a higher dimensionality. The evolution of the boundary of the domain is governed by Hamilton-Jacobi equation. While the shape of the structure may undergo major changes, the level set function remains simple in its topology. Level set methods are numerically efficient and robust procedures for the tracking of interfaces, which allows domain boundary shape changes in the course of iteration. Applications of the level set methods in structural optimization can be found, among others, in [12 2 |14 $|16| 25]$. The speed vector field in Hamilton-Jacobi equation driving the propagation of the level set function is given by the Eulerian derivative of an appropriately defined cost functional with respect to the variations of the free boundary. Recently, in the series of papers [8|9 the level set method employed for the numerical solution of the structural optimization problems are proposed and numerically tested.

This paper deals with topology and shape optimization of elastic contact problems. The optimization problem for elastic contact problem is formulated. Shape as well as topological derivatives formulae of the cost functional are provided using material derivative [20] and asymptotic expansion [22] methods, respectively. These derivatives are employed to formulate necessary optimality condition for simultaneous shape and topology optimization. Level set based numerical algorithm for the solution of the shape optimization problem is proposed. The finite element and finite difference methods [10] are used as the discretization methods. Numerical examples are provided and discussed.

The paper extends the author's previous results in the field of structural optimization of contact systems contained in [15[16] by considering besides shape also topology optimization of these systems or developing optimality conditions, respectively. 


\section{Problem Formulation}

Consider deformations of an elastic body occupying two-dimensional domain $\Omega$ with the smooth boundary $\Gamma$. Assume $\Omega \subset D$ where $D$ is a bounded smooth hold-all subset of $R^{2}$. The body is subject to body forces $f(x)=\left(f_{1}(x), f_{2}(x)\right), x \in \Omega$. Moreover, surface tractions $p(x)=\left(p_{1}(x), p_{2}(x)\right), x \in \Gamma$, are applied to a portion $\Gamma_{1}$ of the boundary $\Gamma$. We assume that the body is clamped along the portion $\Gamma_{0}$ of the boundary $\Gamma$, and that the contact conditions are prescribed on the portion $\Gamma_{2}$, where $\Gamma_{i} \cap \Gamma_{j}=\emptyset, i \neq j, i, j=0,1,2$, $\Gamma=\bar{\Gamma}_{0} \cup \bar{\Gamma}_{1} \cup \bar{\Gamma}_{2}$.

We denote by $u=\left(u_{1}, u_{2}\right), u=u(x), x \in \Omega$, the displacement of the body and by $\sigma(x)=\left\{\sigma_{i j}(u(x))\right\}, i, j=1,2$, the stress field in the body. Consider elastic bodies obeying Hooke's law, i.e., for $x \in \Omega$ and $i, j, k, l=1,2$

$$
\sigma_{i j}(u(x))=a_{i j k l}(x) e_{k l}(u(x)) .
$$

We use here and throughout the paper the summation convention over repeated indices [10]. The strain $e_{k l}(u(x)), k, l=1,2$, is defined by:

$$
e_{k l}(u(x))=\frac{1}{2}\left(u_{k, l}(x)+u_{l, k}(x)\right),
$$

where $u_{k, l}(x)=\frac{\partial u_{k}(x)}{\partial x_{l}}$. The stress field $\sigma$ satisfies the system of equations [10]

$$
-\sigma_{i j}(x)_{, j}=f_{i}(x) \quad x \in \Omega, i, j=1,2,
$$

where $\sigma_{i j}(x)_{, j}=\frac{\partial \sigma_{i j}(x)}{\partial x_{j}}, i, j=1,2$. The following boundary conditions are imposed

$$
\begin{gathered}
u_{i}(x)=0 \text { on } \Gamma_{0}, i=1,2, \\
\sigma_{i j}(x) n_{j}=p_{i} \text { on } \Gamma_{1}, i, j=1,2, \\
u_{N} \leq 0, \sigma_{N} \leq 0, u_{N} \sigma_{N}=0 \text { on } \Gamma_{2}, \\
\left|\sigma_{T}\right| \leq 1, u_{T} \sigma_{T}+\left|u_{T}\right|=0 \text { on } \Gamma_{2},
\end{gathered}
$$

where $n=\left(n_{1}, n_{2}\right)$ is the unit outward versor to the boundary $\Gamma$. Here $u_{N}=u_{i} n_{i}$ and $\sigma_{N}=\sigma_{i j} n_{i} n_{j}, i, j=1,2$, represent the normal components of displacement $u$ and stress $\sigma$, respectively. The tangential components of displacement $u$ and stress $\sigma$ are given by $\left(u_{T}\right)_{i}=u_{i}-u_{N} n_{i}$ and $\left(\sigma_{T}\right)_{i}=\sigma_{i j} n_{j}-\sigma_{N} n_{i}, i, j=1,2$, respectively. $\left|u_{T}\right|$ denotes the Euclidean norm in $R^{2}$ of the tangent vector $u_{T}$. Recall [10], [6] - (7) describe Signorini non-penetration condition and Coulomb friction law, respectively. For the sake of simplicity it is assumed that the tangential contact stress is bounded by 1, i.e., the product of the static friction coefficient and given normal contact stress is equal to 1 . The equality in (7) can be written in the equivalent form as $u_{T} \sigma_{T} \leq 0$ and $\left(1-\left|\sigma_{T}\right|\right) u_{T}=0$. Therefore (7) describes friction phenomenon including sliding. The results concerning the existence of solutions to (1) - (7) can be found in [1020]. 


\subsection{Variational Formulation of Contact Problem}

Let us formulate contact problem (3) - (7) in variational form. Denote by $V_{s p}$ and $K$ the space and set of kinematically admissible displacements:

$$
\begin{gathered}
V_{s p}=\left\{z \in\left[H^{1}(\Omega)\right]^{2}: z_{i}=0 \text { on } \Gamma_{0}, i=1,2\right\}, \\
K=\left\{z \in V_{s p}: z_{N} \leq 0 \text { on } \Gamma_{2}\right\} .
\end{gathered}
$$

$H^{1}(\Omega)$ denotes Sobolev space of square integrable functions and their first derivatives; $\left[H^{1}(\Omega)\right]^{2}=H^{1}(\Omega) \times H^{1}(\Omega)$. Denote also by $\Lambda$ the set

$$
\Lambda=\left\{\zeta \in L^{2}\left(\Gamma_{2}\right):|\zeta| \leq 1\right\} .
$$

Variational formulation of problem (3) - (7) has the form: find a pair $(u, \lambda) \in K \times \Lambda$ satisfying

$$
\begin{gathered}
\int_{\Omega} a_{i j k l} e_{i j}(u) e_{k l}(\varphi-u) d x-\int_{\Omega} f_{i}\left(\varphi_{i}-u_{i}\right) d x-\int_{\Gamma_{1}} p_{i}\left(\varphi_{i}-u_{i}\right) d s \\
+\int_{\Gamma_{2}} \lambda\left(\varphi_{T}-u_{T}\right) d s \geq 0 \quad \forall \varphi \in K, \\
\int_{\Gamma_{2}}(\zeta-\lambda) u_{T} d s \leq 0 \quad \forall \zeta \in \Lambda,
\end{gathered}
$$

$i, j, k, l=1,2$. Function $\lambda$ is interpreted as a Lagrange multiplier corresponding to term $\left|u_{T}\right|$ in equality constraint in (7) [10|20]. This function is equal to tangent stress along the boundary $\Gamma_{2}$, i.e., $\lambda=\sigma_{T_{\mid \Gamma_{2}}}$. Function $\lambda$ belongs to the space $H^{-1 / 2}\left(\Gamma_{2}\right)$, i.e., the space of traces on the boundary $\Gamma_{2}$ of functions from the space $H^{1}(\Omega)$. Here, following [10] function $\lambda$ is assumed to be more regular, i.e., $\lambda \in L^{2}\left(\Gamma_{2}\right)$. The results concerning the existence of solutions to system (10) - (11) can be found, among others, in [10].

\subsection{Optimization Problem}

Before formulating a structural optimization problem for (10) - (11) let us introduce the set $U_{a d}$ of admissible domains. Denote by $\operatorname{Vol}(\Omega)$ the volume of the domain $\Omega$ equal to

$$
\operatorname{Vol}(\Omega)=\int_{\Omega} d x .
$$

Domain $\Omega$ is assumed to satisfy the volume constraint of the form

$$
\operatorname{Vol}(\Omega)-\operatorname{Vol}^{g i v} \leq 0,
$$

where the constant $\mathrm{Vol}^{\text {giv }}=$ const $_{0}>0$ is given. In the case of shape optimization of problem (10) - (11) the optimized domain $\Omega$ is assumed to satisfy equality volume condition, i.e., (13) is assumed to be satisfied as equality. In the case of topology optimization $\mathrm{Vol}^{\text {giv }}$ is assumed to be the initial domain volume and (13) is satisfied in the form $\operatorname{Vol}(\Omega)=r_{f r} \mathrm{Vol}^{g i v}$ with $r_{f r} \in(0,1)$ [22]. The set $U_{a d}$ has the following form

$$
\begin{aligned}
U_{a d}= & \left\{\Omega: E \subset \Omega \subset D \subset R^{2}: \Omega\right. \text { is Lipschitz continuous, } \\
& \left.\Omega \text { satisfies condition (13), } P_{D}(\Omega) \leq \text { const }_{1}\right\},
\end{aligned}
$$


where $E \subset R^{2}$ is a given domain such that $\Omega$ as well as all perturbations of it satisfy $E \subset \Omega . P_{D}(\Omega)=\int_{\Gamma} d x$ is the perimeter of a domain $\Omega$ in $D$ [5], [20, p. 126]. The perimeter constraint is added in (14) to ensure the compactness of the set $U_{a d}$ in the square integrable topology of characteristic functions as well as the existence of optimal domains. The constant const $_{1}>0$ is assumed to exist. The set $U_{a d}$ is assumed to be nonempty. In order to define a cost functional we shall also need the following set $M^{\text {st }}$ of auxiliary functions

$$
M^{s t}=\left\{\phi=\left(\phi_{1}, \phi_{2}\right) \in\left[H^{1}(D)\right]^{2}: \phi_{i} \leq 0 \text { on } D, i=1,2,\|\phi\|_{\left[H^{1}(D)\right]^{2}} \leq 1\right\},
$$

where the norm $\|\phi\|_{\left[H^{1}(D)\right]^{2}}=\left(\sum_{i=1}^{2}\left\|\phi_{i}\right\|_{H^{1}(D)}^{2}\right)^{1 / 2}$.

In order to formulate an optimization problem we have to define the cost functional. Measurements and engineering practice indicate that when two surfaces are in contact a large stress on the contact boundary occurs. Usually, the normal contact stress $\sigma_{N}$ attains maximal values in the middle of the contact area. The goal of structural engineers is to reduce this maximal value of the stress as much as possible. Thus, the cost functional $S\left(\Gamma_{2}\right)=\max _{x \in \Gamma_{2}}\left|\sigma_{N}(x)\right|$ is natural criterion of optimization directly reflecting the design objectives. Unfortunately, the optimization problem with the cost functional $S\left(\Gamma_{2}\right)$ is nonsmooth and difficult for analysis and numerical solution [15]. This is the reason why the criterion of maximal contact stress is approximated by integral, differentiable functionals. Recall from [15] the cost functional approximating the normal contact stress on the contact boundary

$$
J_{\phi}(u(\Omega))=\int_{\Gamma_{2}} \sigma_{N}(u) \phi_{N}(x) d s,
$$

depending on the auxiliary given bounded function $\phi(x) \in M^{s t}$. Function $\phi$ in most cases, including finite-dimensional spaces, is chosen piecewise constant or piecewise linear in a hold-all domain $D$. The integral (16) is nonnegative for all $\phi \in K$. For given $\phi$, the bigger is the normal contact stress on the boundary, the bigger is the value of the cost functional. This integral is also related to the strain energy of the body (for details see [10[15]). $\sigma_{N}$ and $\phi_{N}$ are the normal components of the stress field $\sigma$ corresponding to a solution $u$ satisfying system (10) - 111) and the function $\phi$, respectively.

Consider the following structural optimization problem: for a given function $\phi \in M^{\text {st }}$, find a domain $\Omega^{\star} \in U_{\text {ad }}$ such that

$$
J_{\phi}\left(u\left(\Omega^{\star}\right)\right)=\min _{\Omega \in U_{a d}} J_{\phi}(u(\Omega)) .
$$

The existence of an optimal domain $\Omega^{\star} \in U_{a d}$ follows by standard arguments (see [5|20]).

\section{Optimality Conditions}

\subsection{Shape Derivative}

Consider variations of domain $\Omega \subset D$ with respect to the boundary $\Gamma$ only. Assume that in (14) volume condition is satisfied as equality, i.e., constant volume condition holds. 
Let $\tau$ be a given parameter such that $0 \leq \tau<\tau_{0}, \tau_{0}$ is prescribed, and $V=V(x, \tau), x \in \Omega$, be a given admissible velocity field. The set of admissible velocity fields $V$ consists of vector fields regular enough $\left(C^{k}\right.$ class, $k \geq 1$, for details see [20]) with respect to $x$ and $\tau$ and such that on the boundary $\partial D$ of $D$ either $V=0$ at singular points of this boundary or normal component $V \cdot n$ of $V$ equals $V \cdot n=0$ at points of this boundary where the outward unit normal field $n$ exists. Therefore the perturbations of domain $\Omega$ are governed by the transformation

$$
T(\tau, V): \bar{D} \rightarrow \bar{D},
$$

i.e., $\Omega_{\tau}=T(\tau, V)(\Omega)$ [20]. Since only small perturbations of $\Omega$ are considered, this transformation can have the form of perturbation of the identity operator $I$ in $R^{2}$. An example of such transformation is $T(\tau, \tilde{V})=I+\tau \tilde{V}(x)$, where $\tilde{V}$ denotes a smooth vector field defined on $R^{2}[20]$.

The Euler derivative of the domain functional $J_{\phi}(\Omega)$ is defined as

$$
d J_{\phi}(\Omega, V)=\lim _{\tau \rightarrow 0^{+}} \frac{J_{\phi}\left(\Omega_{\tau}\right)-J_{\phi}(\Omega)}{\tau} .
$$

In [15], using the material derivative approach [20], the Euler derivative of the cost functional (16) has been calculated and a necessary optimality condition for the shape optimization problem (17) has been formulated. This Euler derivative has the form

$$
\begin{aligned}
d J_{\phi}(u(\Omega) ; V) & =\int_{\Gamma}\left(\sigma_{i j} e_{k l}\left(\phi+p^{a d t}\right)-f \cdot \phi\right) V(0) \cdot n d s \\
& -\int_{\Gamma_{1}}\left[\frac{\partial\left(p \cdot\left(p^{a d t}+\phi\right)\right)}{\partial n}+\kappa p \cdot\left(p^{a d t}+\phi\right)\right] V(0) \cdot n d s \\
& +\int_{\Gamma_{2}}\left[\lambda\left(p_{T}^{a d t}+\phi_{T}\right)+q^{a d t} u_{T}\right] \kappa V(0) \cdot n d s,
\end{aligned}
$$

where $i, j, k, l=1,2, V(0)=V(x, 0)$, the displacement $u \in V_{s p}$ and the stress $\lambda \in \Lambda$ satisfy state system (10) - $\kappa$ denotes the mean curvature of the boundary $\Gamma$. The adjoint functions $p^{a d t} \in K_{1}$ and $q^{a d t} \in \Lambda_{1}$ satisfy for $i, j, k, l=1,2$, the following system

$$
\int_{\Omega} a_{i j k l} e_{i j}\left(\phi+p^{a d t}\right) e_{k l}(\varphi) d x+\int_{\Gamma_{2}} q^{a d t} \varphi_{T} d s=0, \forall \varphi \in K_{1}
$$

and

$$
\int_{\Gamma_{2}} \zeta\left(p_{T}^{a d t}+\phi_{T}\right) d s=0, \forall \zeta \in \Lambda_{1}
$$

where the cones $K_{1}$ and $\Lambda_{1}$ are given by [15]20]

$$
\begin{gathered}
K_{1}=\left\{\xi \in V_{s p}: \xi_{N}=0 \text { on } A^{s t}\right\}, \\
\Lambda_{1}=\left\{\zeta \in L^{2}\left(\Gamma_{2}\right): \zeta(x)=0 \text { on } B_{1} \cup B_{2} \cup B_{1}^{+} \cup B_{2}^{+}\right\},
\end{gathered}
$$

while the coincidence set $A^{s t}=\left\{x \in \Gamma_{2}: u_{N}=0\right\}$. Moreover $B_{1}=\left\{x \in \Gamma_{2}: \lambda(x)=-1\right\}$, $B_{2}=\left\{x \in \Gamma_{2}: \lambda(x)=+1\right\}, \tilde{B}_{i}=\left\{x \in B_{i}: u_{N}(x)=0\right\}, i=1,2, B_{i}^{+}=B_{i} \backslash \tilde{B}_{i}, i=1,2$. The necessary optimality condition is formulated in [15].

Lemma 1. Let $\Omega^{\star} \in U_{a d}$ be an optimal solution to the problem (17). Then there exist Lagrange multipliers $\mu_{1} \in R$, associated with the constant volume constraint and $\mu_{2} \in$ $R, \mu_{2} \geq 0$, associated with the finite perimeter constraint such that for all admissible 
vector fields $V$ and such that all perturbations $\delta \Omega \in U_{\text {ad }}$ of domain $\Omega \in U_{\text {ad }}$ satisfy $E \subset \Omega \cup \delta \Omega \subset D$, at any optimal solution $\Omega^{\star} \in U_{\text {ad }}$ to the shape optimization problem (17) the following conditions are satisfied:

$$
\begin{gathered}
d J_{\phi}\left(u\left(\Omega^{\star}\right) ; V\right)+\mu_{1} \int_{\Gamma^{\star}} V(0) \cdot n d s+\mu_{2} d P_{D}\left(\Omega^{\star} ; V\right) \geq 0, \\
\mu_{1}\left(\int_{\Omega^{\star}} d x-\text { const }_{0}\right)=0,\left(\mu_{2}^{\sim}-\mu_{2}\right)\left(P_{D}\left(\Omega^{\star}\right)-\text { const }_{1}\right) \leq 0, \\
\forall \mu_{2}^{\sim} \in R, \mu_{2}^{\sim} \geq 0,
\end{gathered}
$$

where $u\left(\Omega^{\star}\right)$ denotes the solution to (10) - (11) in the domain $\Omega^{\star}, \Gamma^{\star}=\partial \Omega^{\star}$, the Euler derivative $d J_{\phi}\left(u\left(\Omega^{\star}\right) ; V\right)$ is given by (19) and $d P_{D}(\Omega ; V)$ denotes the Euler derivative of the finite perimeter functional $P_{D}(\Omega)$ (see [20 p. 126]). The given constant const $t_{0}>$ 0 and constant const $1>0$ are the same as in (14).

\subsection{Topological Derivative}

Classical shape optimization is based on the perturbation of the boundary of the initial shape domain. The initial and final shape domains have the same topology. The aim of the topological optimization is to find an optimal shape without any a priori assumption about the structure's topology.

The value of the goal functional (16) can be minimized by the topology variation of the domain $\Omega$. The topology variations of geometrical domains are defined as functions of a small parameter $\rho$ such that $0<\rho<R, R>0$ given. They are based on the creation of a small hole

$$
B(x, \rho)=\left\{z \in R^{2}:|x-z|<\rho\right\}
$$

of radius $\rho$ at a point $x \in \Omega$ in the interior of the domain $\Omega$. The Neumann boundary conditions are prescribed on the boundary $\partial B$ of the hole. Denote the perturbed domain by $\Omega_{\rho}=\Omega \backslash \overline{B(x, \rho)}$.

The topological derivative $T J_{\phi}(\Omega, x)$ of the domain functional $J_{\phi}(\Omega)$ at $\Omega \subset R^{2}$ is a function depending on a center $x$ of the small hole and is defined by [1]17/22]

$$
T J_{\phi}(\Omega, x)=\lim _{\rho \rightarrow 0^{+}}\left[J_{\phi}(\Omega \backslash \overline{B(x, \rho)})-J_{\phi}(\Omega)\right] / \pi \rho^{2} .
$$

This derivative is calculated by the asymptotic expansion method [22]. To minimize the cost functional $J_{\phi}(\Omega)$ the holes have to be created at the points of domain $\Omega$ where $T J_{\phi}$ is negative.

The formulae for topological derivatives of cost functionals for plane elasticity systems or contact problems are provided, among others, in papers [6/7 21 23]. Using the methodology from [22] as well as the results of differentiability of solutions to variational inequalities [20], we can calculate the formulae of the topological derivative $T J_{\phi}\left(\Omega ; x_{0}\right)$ of the cost functional $\left[16\right.$ at a point $x_{0} \in \Omega$. This derivative is equal to

$$
\begin{aligned}
T J_{\phi}\left(u(\Omega), x_{0}\right) & =-\left[f\left(\phi+w^{a d t}\right)+\frac{1}{E}\left(a_{u} a_{w^{a d t}+\phi}+2 b_{u} b_{w^{a d t}+\phi} \cos 2 \delta\right)\right]_{\mid x=x_{0}} \\
& -\int_{\Gamma_{2}}\left(s^{a d t} u_{T}+\lambda\left(w_{T}^{a d t}+\phi_{T}\right)\right) \kappa d s,
\end{aligned}
$$


where $a_{\tilde{\beta}}=\sigma_{I}(\tilde{\beta})+\sigma_{I I}(\tilde{\beta}), b_{\tilde{\beta}}=\sigma_{I}(\tilde{\beta})-\sigma_{I I}(\tilde{\beta})$, and either $\tilde{\beta}="$ " " or $\quad \tilde{\beta}=$ "wadt $+\phi ", \sigma_{I}(u)$ and $\sigma_{I I}(u)$ denote principal stresses for displacement $u, \delta$ is the angle between principal stresses directions [22]. E denotes Young modulus.

In order to obtain formula (29), the plane elasticity system (3) - (5) is written in the polar coordinate system aligned with the principal stress directions. The asymptotic expansions of displacement, strain and stress with respect to parameter $\rho$ in the ring adjacent to the hole $B(x, \rho)$ hold [21]. Taking into account these asymptotic expansions, the regularity of solutions to the state system (3) - (7), calculating the derivatives of the cost functional (16) and solutions to the state system (3) - (7) with respect to parameter $\rho$ as well as using the results of differentiability of solutions to variational inequalities we obtain 29). The integral term in 29) follows from the assumption that tangent displacement and stress functions along $\Gamma_{2}$ are dependent on the parameter $\rho$.

The adjoint state $\left(w_{\rho}^{a d t}, s_{\rho}^{a d t}\right) \in K_{1} \times \Lambda_{1}$ satisfies system (20) - 21) in domain $\Omega_{\rho}$ rather than $\Omega$, i.e.,

$$
\int_{\Omega_{\rho}} a_{i j k l} e_{i j}\left(\phi+w_{\rho}^{a d t}\right) e_{k l}(\varphi) d x+\int_{\Gamma_{2}} s_{\rho}^{a d t} \varphi_{T} d s=0, \forall \varphi \in K_{1},
$$

and

$$
\int_{\Gamma_{2}} \zeta\left(w_{\rho T}^{a d t}+\phi_{T}\right) d s=0, \quad \forall \zeta \in \Lambda_{1},
$$

where $w_{\rho}^{a d t}{ }_{\mid \rho=0}=w^{a d t}\left(x_{0}\right)$. By standard arguments [52021] it can be shown that if $\Omega^{\star} \in U_{a d}$ is an optimal domain to the problem (17) it satisfies for all $x_{0} \in \Omega^{\star}$ the necessary optimality condition of the form (24) - 26) with topological derivative 29] rather than Euler derivative (19) in (24), and inequality in 25) rather than equality as well as with Lagrange multiplier $\mu_{1} \geq 0$.

\subsection{Domain Differential}

Finally consider the variation of the functional (16) resulting both from the nucleation of the internal small hole as well as from the boundary variations. In order to take into account these perturbations, in [21] the notion of the domain differential of the domain functional has been introduced. The domain differential $D J_{\phi}\left(\Omega ; V, x_{0}\right)$ of the shape functional (16) at $\Omega \subset R^{2}$ in direction $V$ and at point $x_{0} \in \Omega$ is defined as

$$
D J_{\phi}\left(\Omega ; V, x_{0}\right)(\tau, \rho)=\tau d J_{\phi}(\Omega, V)+\pi \rho^{2} T J_{\phi}\left(\Omega, x_{0}\right) .
$$

This differential completely characterizes the variation of the cost functional $J_{\phi}(\Omega)$ with respect to the simultaneous shape and topology perturbations (for details see [21]). The shape derivative $d J_{\phi}(u(\Omega), V)$ and the topological derivative $T J_{\phi}\left(u(\Omega), x_{0}\right)$ are provided by (19) and (29) respectively. They depend on the solution $u$ to the state system [10] - [11]. Using standard arguments [20] we can show

Lemma 2. Let $\Omega^{\star} \in U_{a d}$ be an optimal solution to the problem (17). Then there exist Lagrange multipliers $\mu_{1} \in R, \mu_{1} \geq 0$, associated with the volume constraint and $\mu_{2} \in R$, $\mu_{2} \geq 0$, associated with the finite perimeter constraint such that for all admissible vector 
fields $V$, for all admissible pairs $(\rho, \tau)$ of parameters and for all $x_{0} \in \Omega^{\star}$ and such that all perturbations $\delta \Omega \in U_{\text {ad }}$ of domain $\Omega \in U_{\text {ad }}$ satisfy $E \subset \Omega \cup \delta \Omega \subset D$, at any optimal solution $\Omega^{\star} \in U_{\text {ad }}$ to the shape and topology optimization problem (17) the following conditions are satisfied:

$$
\begin{gathered}
D J_{\phi}\left(u\left(\Omega^{\star}\right) ; V, x_{0}\right)(\tau, \rho)+\mu_{1} \int_{\Gamma^{\star}} V(0) \cdot n d s+\mu_{2} d P_{D}\left(\Omega^{\star} ; V\right) \geq 0, \\
\left(\mu_{1}^{\sim}-\mu_{1}\right)\left(\int_{\Omega^{\star}} d x-\text { const }_{0}\right) \leq 0, \forall \mu_{1}^{\sim} \in R, \mu_{1}^{\sim} \geq 0, \\
\left(\mu_{2}^{\sim}-\mu_{2}\right)\left(P_{D}\left(\Omega^{\star}\right)-\text { const }_{1}\right) \leq 0, \forall \mu_{2}^{\sim} \in R, \mu_{2}^{\sim} \geq 0,
\end{gathered}
$$

where $u\left(\Omega^{\star}\right)$ denotes the solution to (10) - (11) in the domain $\Omega^{\star}, \Gamma^{\star}=\partial \Omega^{\star}$, the domain differential $D J_{\phi}\left(u\left(\Omega^{\star}\right) ; V, x_{0}\right)(\tau, \rho)$ is given by (32) and $d P_{D}(\Omega ; V)$ denotes the derivative of the finite perimeter functional $P_{D}(\Omega)$ (see [1,6],[20, p. 126]). The given constant const $t_{0}>0$ and constant const $t_{1}>0$ are the same as in (14).

\section{Shape Representation by Level Set Method}

In the paper the level set method [19] is employed to solve numerically problem (17). Consider the evolution of a domain $\Omega$ under a velocity field $V$. Let $t>0$ denote the time variable. Under the mapping $T(t, V)$ we have

$$
\Omega_{t}=T(t, V)(\Omega)=(I+t V)(\Omega), t>0 .
$$

By $\Omega_{t}^{-}$we denote the interior of the domain $\Omega_{t}$ and by $\Omega_{t}^{+}$we denote the outside of the domain $\Omega_{t}$. The domain $\Omega_{t}$ and its boundary $\partial \Omega_{t}$ are defined by a function $\Phi=\Phi(x, t): R^{2} \times\left[0, t_{0}\right) \rightarrow R$ satisfying

$$
\left\{\begin{array}{l}
\Phi(x, t)=0, \text { if } x \in \partial \Omega_{t}, \\
\Phi(x, t)<0, \text { if } x \in \Omega_{t}^{-}, \\
\Phi(x, t)>0, \text { if } x \in \Omega_{t}^{+},
\end{array}\right.
$$

i.e., the boundary $\partial \Omega_{t}$ is the level curve of the function $\Phi$. Recall [19], the gradient of the implicit function is defined as $\nabla \Phi=\left(\frac{\partial \Phi}{\partial x_{1}}, \frac{\partial \Phi}{\partial x_{2}}\right)$, the local unit outward normal $n$ to the boundary is equal to $n=\frac{\nabla \Phi}{|\nabla \Phi|}$, the mean curvature $\kappa=\nabla \cdot n$. In the level set approach, Heaviside function $H(\Phi)$ and Dirac function $\delta(\Phi)$ are used to transform integrals from domain $\Omega$ into domain $D$. These functions are defined as

$$
\begin{gathered}
H(\Phi)=1 \text { if } \Phi \geq 0, \quad H(\Phi)=0 \text { if } \Phi<0, \\
\delta(\Phi)=H^{\prime}(\Phi), \delta(x)=\delta(\Phi(x))|\nabla \Phi(x)|, x \in D .
\end{gathered}
$$

Assume that velocity field $V$ is known for every point $x$ lying on the boundary $\partial \Omega_{t}$, i.e., with $\Phi(x, t)=0$. Therefore the equation governing the evolution of the interface in $D \times\left[0, t_{0}\right]$ has the form [19]

$$
\Phi_{t}(x, t)+V(x, t) \cdot \nabla_{x} \Phi(x, t)=0,
$$

where $\Phi_{t}$ denotes a partial derivative of $\Phi$ with respect to the time variable $t$. Equation (40) is known as Hamilton-Jacobi equation. 


\subsection{Structural Optimization Problem in Domain $D$}

Using the notion of the level set function (37) as well as functions (38) and 39) structural optimization problem (17) may be reformulated in the following way: for a given function $\phi \in M^{\text {st }}$, find function $\Phi$ such that

$$
J_{\phi}\left(u\left(\Phi^{\star}\right)\right)=\min _{\Phi \in U_{a d}^{\Phi}} J_{\phi}(u(\Phi))
$$

where

$$
\begin{gathered}
J_{\phi}(u(\Phi))=\int_{D} \sigma_{N}(u) \phi_{N}(x) \delta(\Phi)|\nabla \Phi| d s, \\
U_{a d}^{\Phi}=\left\{\Phi: \Phi \text { satisfies } \underline{37}, \operatorname{Vol}(\Phi) \leq \operatorname{Vol}^{\text {giv }}, P_{D}(\Phi) \leq \text { const }_{1}\right\}, \\
\operatorname{Vol}(\Phi)=\int_{D} H(\Phi) d x, \\
P_{D}(\Phi)=\int_{D} \delta(\Phi)|\nabla \Phi| d x .
\end{gathered}
$$

Moreover, a pair $(u, \lambda) \in K \times \Lambda$ satisfies system

$$
\begin{gathered}
\int_{D} a_{i j k l} e_{i j}(u) e_{k l}(\varphi-u) H(\Phi) d x-\int_{D} f_{i}\left(\varphi_{i}-u_{i}\right) H(\Phi) d x \\
-\int_{D} p_{i}\left(\varphi_{i}-u_{i}\right) \delta(\Phi)|\nabla \Phi| d x \\
+\int_{D} \lambda\left(\varphi_{T}-u_{T}\right) \delta(\Phi)|\nabla \Phi| d x \geq 0 \forall \varphi \in K, \\
\int_{D}(\zeta-\lambda) u_{T} \delta(\Phi)|\nabla \Phi| d x \leq 0 \forall \zeta \in \Lambda,
\end{gathered}
$$

while $V_{s p}$ and $K$ are defined by (8) and (9), respectively, on domain $D$ rather than $\Omega$ and $i, j, k, l=1,2$.

\section{Level Set Based Numerical Algorithm}

The topological derivative can provide better prediction of the structure topology with different levels of material volume than the method based on updating the shape of initial structure containing many regularly distributed holes [1|25]. Our approach is based on the application of the topological derivative to predict the structure topology and substitute material according to the material volume constraint and next to optimize the structure topology to merge the unreasonable material interfaces and to change the shape of material boundary. For the sake of simplicity in the description of the algorithm we omit the bounded perimeter constraint in (14). Therefore the level set method combined with the shape or topological derivatives results in the following conceptual algorithm (A1) to solve structural optimization problem (17):

Step 1: Choose: a computational domain $D$ such that $\Omega \subset D$, an initial level set function $\Phi^{0}=\Phi_{0}$ representing $\Omega^{0}=\Omega$, function $\phi \in M^{s t}$, parameters $r^{0}, \varepsilon_{1}, \varepsilon_{2}, \tilde{\mu}_{1}^{0}=$ $\mu_{1}^{0}=0, k=n=0$.

Step 2: Calculate the solution $\left(u^{n}, \lambda^{n}\right)$ to the state system 46 - 47). 
Step 3: Calculate the solution $\left(\left(w^{a d t}\right)^{n},\left(s^{a d t}\right)^{n}\right)$ to the adjoint system 30 - 31 as well as the topological derivative $T J_{\phi}\left(\Omega^{n}, x\right)$ of the cost functional (16) given by (29).

Step 4: For given $\tilde{\mu}_{1}^{n}$ set $\Omega^{n+1}=\left\{x \in \Omega^{n}: T J_{\phi}(\Omega, x) \geq \chi_{n+1}\right\}$ where $\chi_{n+1}$ is chosen in such a way that $\operatorname{Vol}\left(\Omega^{n+1}\right)=m_{n+1}, m_{n+1}=q m_{n}$. Fill the void part $D \backslash \Omega^{n+1}$ with a very weak material with Young modulus $E^{w}=10^{-5} E$. Update $\tilde{\mu}_{1}^{n+1}=\tilde{\mu}_{1}^{n}+$ $r^{n}\left(\operatorname{Vol}_{1}^{\text {giv }}\right), r^{n}>0, \operatorname{Vol}_{1}^{\text {giv }}=\operatorname{Vol}\left(\Omega^{n+1}\right)-r_{f r} \operatorname{Vol}^{\text {giv }}$. If $\left|\tilde{\mu}_{1}{ }^{n+1}-\tilde{\mu}_{1}{ }^{n}\right| \leq \varepsilon_{1}$ then set $\Omega^{k}=\Omega^{n+1}$ and go to Step 5. Otherwise set $n=n+1$, go to Step 2 .

Step 5: Calculate the solution $\left(\left(p^{a d t}\right)^{k},\left(q^{a d t}\right)^{k}\right)$ to the adjoint system 201 - 21). Calculate the shape derivative $d J_{\phi}\left(u\left(\Omega^{k}\right)\right)$ of the cost functional (16) given by (19).

Step 6: For given $\mu_{1}^{k}$ solve the level set equation (40) to calculate the level set function $\Phi^{k+1}$.

Step 7: Set $\Omega^{k+1}$ equal to the zero level set of function $\Phi^{k+1}$. Calculate $\mu_{1}^{k+1}=\mu_{1}^{k}+$ $r^{k}\left(\operatorname{Vol}\left(\Omega^{k+1}\right)-\operatorname{Vol}_{1}^{g i v}\right), r^{k}>0$. If $\left|\mu_{1}^{k+1}-\mu_{1}^{k}\right| \leq \varepsilon_{2}$ then Stop. Otherwise set $k=k+1, \Omega^{n}=\Omega^{k+1}$, and go to Step 2 .

Let us remark that having localized a small hole one can consider to perform further the size optimization of the radius of the existing hole rather than shape optimization. However such approach, confining to hole change only inside the optimized domain and not allowing for the change of the shape of the external boundary of the body seems to be not versatile. Level set approach allowing for tracking changes of internal and external interfaces of the optimized domain on a fixed mesh, including the merging of holes, seems to be more suitable in simultaneous shape and topology optimization of domains.

State (10) - 111) and adjoint 201 - 21) systems are discretized using finite element method [10]. Displacement and stress functions in state system (10) - (11) are approximated by piecewise bilinear functions in domain $D$ and piecewise constant functions on the boundary $\Gamma_{2}$ respectively. Similar approximation is used to discretize the adjoint system (20) - 21) or (30) - 231). These systems are solved using the primal-dual algorithm with active set strategy [3|24]. In level set approach these state and adjoint systems are transferred from domain $\Omega$ into fixed hold-all domain $D$ using the regularized Heaviside and Dirac functions. Finite difference method is employed to discretize Hamilton-Jacobi equation and the explicit up-wind scheme is used to solve it. For more details concerning implementation of this algorithm see [16].

\section{Numerical Methods and Example}

The discretized structural optimization problem (17) is solved numerically. The numerical algorithms described in the previous sections have been used. The algorithm is programmed in Matlab environment. As an example a body occupying 2D domain

$$
\Omega=\left\{\left(x_{1}, x_{2}\right) \in R^{2}: 0 \leq x_{1} \leq 8 \wedge 0<v\left(x_{1}\right) \leq x_{2} \leq 4\right\},
$$

is considered. The boundary $\Gamma$ of the domain $\Omega$ is divided into three pieces

$$
\begin{gathered}
\Gamma_{0}=\left\{\left(x_{1}, x_{2}\right) \in R^{2}: x_{1}=0,8 \wedge 0<v\left(x_{1}\right) \leq x_{2} \leq 4\right\}, \\
\Gamma_{1}=\left\{\left(x_{1}, x_{2}\right) \in R^{2}: 0 \leq x_{1} \leq 8 \wedge x_{2}=4\right\}, \\
\Gamma_{2}=\left\{\left(x_{1}, x_{2}\right) \in R^{2}: 0 \leq x_{1} \leq 8 \wedge v\left(x_{1}\right)=x_{2}\right\} .
\end{gathered}
$$




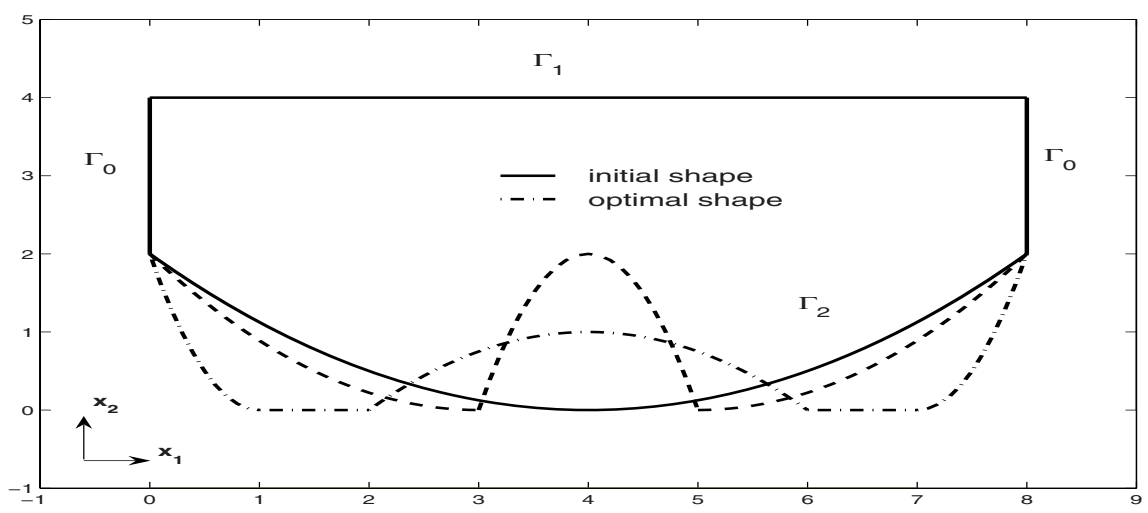

Fig. 1. Shape optimization - optimal domain

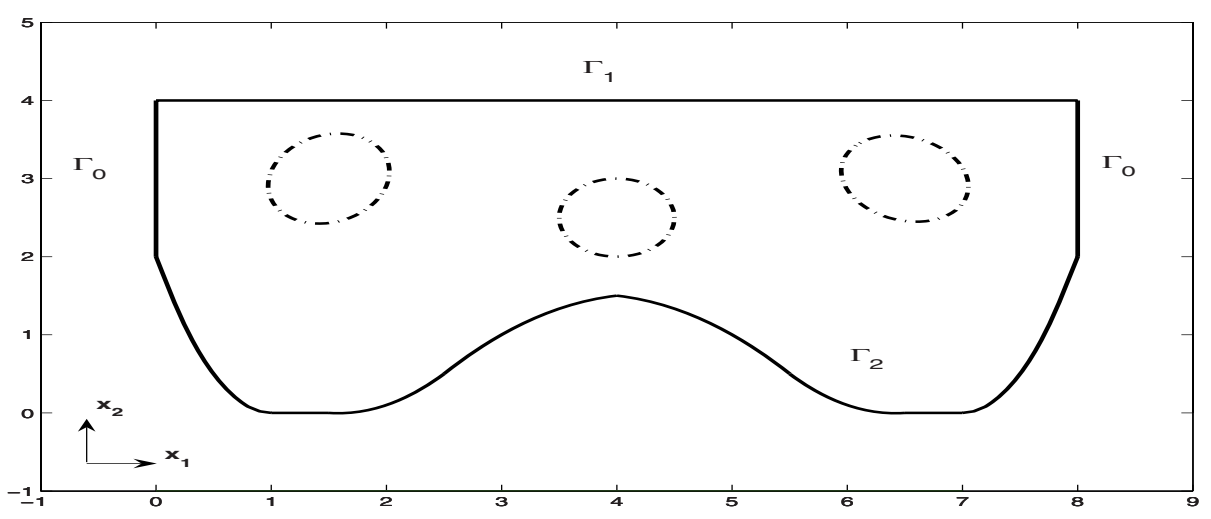

Fig. 2. Simultaneous topology and shape optimization - optimal domain

The domain $\Omega$ and the boundary $\Gamma_{2}$ depend on the function $v$. This function is the variable subject to shape optimization [13]15[16]. The initial position of the boundary $\Gamma_{2}$ is given as in Fig. 1. The computations are carried out for the elastic body characterized by the Poisson's ratio $v=0.29$, the Young modulus $E=2.1 \cdot 10^{11} \mathrm{~N} / \mathrm{m}^{2}$. The body is loaded by boundary traction $p_{1}=0, p_{2}=-5.6 \cdot 10^{6} \mathrm{~N}$ along $\Gamma_{1}$, body forces $f_{i}=0$, $i=1,2$. Auxiliary function $\phi$ is selected as piecewise constant (or linear) on $D$ and is approximated by a piecewise constant (or bilinear) functions. The computational domain $D=[0,8] \times[0,4]$ is selected. Domain $D$ is discretized with a fixed rectangular mesh of $24 \times 12$.

Fig. 1 displays the optimal solution of shape optimization problem (17). Fig. 2 presents the optimal domain obtained by solving topological and shape optimization problem (17) in the computational domain $D$ using algorithm (A1) and employing the optimality condition (33) - 35). The areas of holes denoted by dotted lines appear in the central part of the body and near the fixed edges. These areas result from the merging of small holes from which the material has been removed into the bigger ones. Recall that 
the application of formula 29) allows to insert infinitesimal holes only. Although the shape of the optimal contact boundary $\Gamma_{2}$ is similar to the optimal shape obtained in the case of shape optimization only (see Fig. 2) but the obtained shape of the boundary $\Gamma_{2}$ is not so strongly changed comparing to the initial one as the optimal shape obtained in the case of shape optimization only. The obtained normal contact stress is almost constant along the optimal shape boundary and has been significantly reduced comparing to the initial one.

\section{Conclusions}

The structural optimization problem for elastic contact problem with the prescribed friction is solved numerically in the paper. The topological derivative method as well as the level set approach combined with the shape gradient method are used. The friction term complicates both the form of the gradients of the cost or penalty functionals as well as numerical process.

Obtained preliminary numerical results seem to be in accordance with physical reasoning. They indicate that the proposed numerical algorithm allows for significant improvements of the structure from one iteration to the next. They also indicate the future research direction aiming at better reconciliation in one algorithm holes nucleation flexibility and geometric update fidelity.

\section{References}

1. Allaire, G., Jouve, F., Toader, A.: Structural optimization using sensitivity analysis and a level set method. Journal of Computational Physics 194(1), 363-393 (2004)

2. Burger, M., Hackl, B., Ring, W.: Incorporating topological derivatives into level set methods. Journal of Computational Physics 194(1), 344-362 (2004)

3. Bergonioux, M., Kunisch, K.: Augmented Lagrangian techniques for elliptic state constrained optimal control problems. SIAM Journal on Control and Optimization 35, 1524 1543 (1997)

4. Chopp, H., Dolbow, J.: A hybrid extended finite element / level set method for modelling phase transformations. International Journal for Numerical Methods in Engineering 54, 1209-1232 (2002)

5. Delfour, M., Zolesio, J.-P.: Shapes and Geometries: Analysis, Differential Calculus and Optimization. SIAM Publications, Philadelphia (2001)

6. Fulmański, P., Laurain, A., Scheid, J.F., Sokołowski, J.: A Level Set Method in Shape and Topology Optimization for Variational Inequalities. Int. J. Appl. Math. Comput. Sci. 17, 413430 (2007)

7. Garreau, S., Guillaume, Ph., Masmoudi, M.: The topological asymptotic for PDE systems: the elasticity case. SIAM Journal on Control Optimization 39, 1756-1778 (2001)

8. Gomes, A., Suleman, A.: Application of spectral level set methodology in topology optimization. Structural Multidisciplinary Optimization 31, 430-443 (2006)

9. de Gourmay, F.: Velocity extension for the level set method and multiple eigenvalue in shape optimization. SIAM Journal on Control and Optimization 45(1), 343-367 (2006)

10. Haslinger, J., Mäkinen, R.: Introduction to Shape Optimization. Theory, Approximation, and Computation. SIAM Publications, Philadelphia (2003) 
11. He, L., Kao, Ch.Y., Osher, S.: Incorporating topological derivatives into shape derivatives based level set methods. Journal of Computational Physics 225, 891-909 (2007)

12. Hintermüller, M., Ring, W.: A level set approach for the solution of a state-constrained optimal control problem. Numerische Mathematik 98, 135-166 (2004)

13. Myśliński, A.: Level set method for shape optimization of contact problems. In: Neittaanmäki, P., Jyväskylä. (eds.) Proceedings of European Congress on Computational Methods in Applied Sciences and Engineering, Finland (2004)

14. Myśliński, A.: Topology and shape optimization of contact problems using a level set method. In: Herskovits, J., Mazorche, S., Canelas, A. (eds.) Proceedings of VI World Congresses of Structural and Multidisciplinary Optimization, Rio de Janeiro, Brazil (2005)

15. Myśliński, A.: Shape Optimization of Nonlinear Distributed Parameter Systems. Academic Printing House EXIT, Warsaw (2006)

16. Myśliński, A.: Level Set Method for Optimization of Contact Problems. Engineering Analysis with Boundary Elements 32, 986-994 (2008)

17. Novotny, A.A., Feijóo, R.A., Padra, C., Tarocco, E.: Topological derivative for linear elastic plate bending problems. Control and Cybernetics 34(1), 339-361 (2005)

18. Norato, J.A., Bendsoe, M.P., Haber, R., Tortorelli, D.A.: A topological derivative method for topology optimization. Structural Multidisciplinary Optimization 33, 375-386 (2007)

19. Osher, S., Fedkiw, R.: Level Set Methods and Dynamic Implicit Surfaces. Springer, New York (2003)

20. Sokołowski, J., Zolesio, J.-P.: Introduction to Shape Optimization. Shape Sensitivity Analysis. Springer, Berlin (1992)

21. Sokołowski, J., Żochowski, A.: Optimality conditions for simultaneous topology and shape optimization. SIAM Journal on Control 42(4), 1198-1221 (2003)

22. Sokołowski, J., Żochowski, A.: On topological derivative in shape optimization. In: Lewiński, T., Sigmund, O., Sokołowski, J., Żochowski, A. (eds.) Optimal Shape Design and Modelling, pp. 55-143. Academic Printing House EXIT, Warsaw (2004)

23. Sokołowski, J., Żochowski, A.: Modelling of topological derivatives for contact problems. Numerische Mathematik 102(1), 145-179 (2005)

24. Stadler, G.: Semismooth Newton and augmented Lagrangian methods for a simplified friction problem. SIAM Journal on Optimization 15(1), 39-62 (2004)

25. Wang, M.Y., Wang, X., Guo, D.: A level set method for structural topology optimization. Computer Methods in Applied Mechanics and Engineering 192, 227-246 (2003)

26. Xia, Q., Wang, M.Y., Wang, S., Chen, S.: Semi-Lagrange method for level set based structural topology and shape optimization. Multidisciplinary Structural Optimization 31, 419429 (2006)

27. Wang, S.Y., Lim, K.M., Khao, B.C., Wang, M.Y.: An extended level set method for shape and topology optimization. Journal of Computational Physics 221, 395-421 (2007) 